Erschienen in: Lang, Ewald/Zifonun, Gisela (Hrsg.): Deutsch - typologisch. Berlin,

New York: de Gruyter, 1996. S. 359-368.

(Jahrbuch des Instituts für deutsche Sprache 1995)

DOI: https://doi.org/10.1515/9783110622522-016

\title{
ÖSTEN DAHL
}

\section{Das Tempussystem des Deutschen im typologischen Vergleich}

\begin{abstract}
Als grundlegende Analyseeinheit für das typologische Studium von Tempusund Aspektsystemen wird das 'Gramm' eingeführt - eine Kategorie, unter die zum Beispiel 'Progressiv' oder 'Perfektiv' fallen. Im typologischen Vergleich spielen übereinzelsprachliche Grammtypen eine Rolle. Bezüglich der Verteilung von Grammtypen lassen sich in den europäischen Sprachen bestimmte areale Tendenzen feststellen. Das deutsche Tempus- und Aspektsystem kann als relativ "arm” eingeordnet werden. Genauer analysiert werden das Perfekt und die Ausdrucksmöglichkeiten für Zukunft im Deutschen.
\end{abstract}

\section{Typologisches über Tempus und Aspekt}

Das typologische Studium von Tempus- und Aspektsystemen ist nicht so gut entwickelt wie z.B. die Wortfolgetypologie. Das hängt wohl damit zusammen, daß es sich in diesem Bereich um schwer vergleichbare, semantisch definierte Kategorien handelt. Doch sollten Arbeiten wie Friedrich (1974), Comrie (1976), (1985), Chung/Timberlake (1985), Ultan (1978) erwähnt werden.

Ende der siebziger Jahre habe ich eine datenorientierte Untersuchung von Tempus- und Aspektsystemen verschiedener Sprachen initiiert. Mit Hilfe eines Fragebogens von etwa 160 Sätzen, die aus dem Englischen in die jeweilige Sprachen übersetzt wurden, haben wir Materialien aus 64 Sprachen gesammelt, die eine Art „convenience sample” ausmachten. Zu derselben Zeit haben Joan Bybee und ihre Mitarbeiter Revere Perkins und William Pagliuca eine Untersuchung von morphologischen Verbkategorien in einer kontrollierten Auswahl von 50 Sprachen durchgeführt, mit Hilfe existierender grammatischer Beschreibungen als hauptsächlicher Informationsquelle. Die Ergebnisse dieser Projekte wurden gleichzeitig, aber unabhängig voneinander publiziert, in den Monographien Dahl (1985) bzw. Bybee (1985), und waren trotz der unterschiedlichen Methodologie, auffallend ähnlich. 1989 haben wir einen gemeinsamen Artikel verfaßt, in dem wir versuchten, unsere Ansätze zu integrieren. Vor kurzem haben Bybee, Perkins und Pagliuca ihre gemeinsame Arbeit in einer Monographie vorgelegt (Bybee et al. 1994). Dieses Buch ist zur Zeit das vollständigste und aktuellste Werk über Tempus, Aspekt und Modalität aus einer typologischen Perspektive. 


\section{"Gramme"}

Die grundlegende Analyseeinheit in Bybee/Dahl (1989) und Bybee et al. (1994) sind nicht die traditionellen Kategorien Tempus und Aspekt sondern, was wir grams, zu Deutsch "Gramme”, nennen d.h. Kategorien wie das Progressiv im Englischen, das Passé simple im Französischen usw. Begriffe wie Tempus, Modus und Aspekt sehen wir als Charakterisierungen zu dem semantischen Inhalt eines Gramms, oder lieber als Domäne, aus denen ihre Bedeutungen gewählt werden. Im typischen Fall sind sie aber nicht strukturell wesentliche Einheiten grammatischer Systeme. Viele, vielleicht die meisten Gramme verbinden in ihrer Semantik Elemente aus verschiedenen Domänen, und es ist eher die Regel als eine Ausnahme, daB Gramme, die traditionell unter einer Kategorie geführt worden sind, im Hinblick auf ihren Ausdruck sehr unterschiedlich sind.

Der Begriff Gramm ist auf der sprachspezifischen Ebene zu verstehen, d.h. ein Gramm gehört zur Grammatik einer bestimmten Sprache eher als zur allgemeinen Grammatiktheorie. Eine wichtige These unserer Arbeiten ist jedoch, daB Tempus- und Aspektgramme unter eine relativ begrenzte Zahl von übereinzelsprachlichen Typen fallen. In der universellen Grammatiktheorie ist also die grundlegende Einheit der übereinzelsprachliche Gramm-Typ, der in den individuellen Sprachen als Gramm manifestiert wird. Diese Grammtypen sollen nicht als absolute Einheiten - etwa als Buchstaben eines universalen Alphabets - verstanden werden, sondern als die statistisch wahrscheinlichsten Bündelungen von Eigenschaften im "grammatischen Raum" oder als vergleichmäBig stabile Punkte längs der Grammatikalisierungswege, die die Gramme in ihrer Entwicklung beschreiben. Die Grammtypen sind auch nicht „semantische Kategorien” oder "Begriffskategorien”. Freilich ist ein Grammtyp von seiner Semantik zusammengehalten, aber es ist doch wesentlich, daß ein Grammtyp nicht ein Begriff ist, sondern ein Typ von grammatischem Element, das auch im Hinblick auf seine Ausdruckseigenschaften charakterisiert werden kann: jeder Grammtyp hat eine typische Ausdrucksweise, die seiner Position in den Grammatikalisierungsprozessen entspricht.

Ich habe auch den Begriff Grammfamilie (gram family) eingeführt. Eine Grammfamilie ist eine Gruppe von Grammen, die in einem historischen Prozeß erstanden sind - entweder durch Erbe von einer gemeinsamen Ursprache oder durch Sprachkontakt. 


\section{Die Struktur eines typischen Tempus- und Aspektsystems}

In Figur 1 werden die wichtigsten Tempus- und Aspekt-Grammtypen dargestellt. Die Pfeile zeigen die möglichen Grammatikalisierungsprozesse.

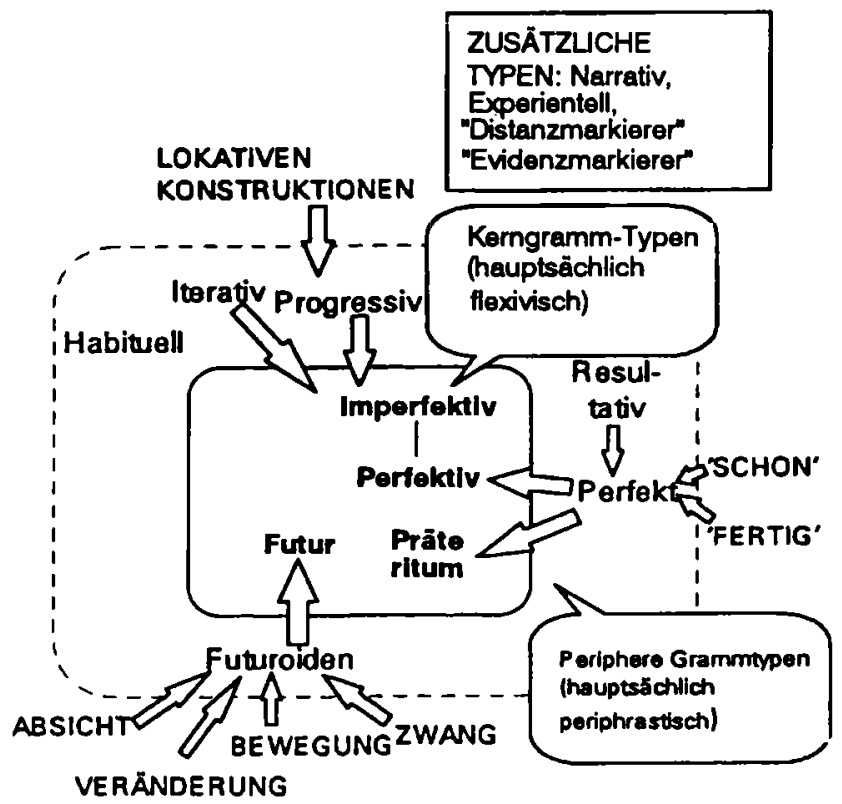

Figur 1. Hauptgrammtypen

Als Glieder des "harten Kerns” von Tempus- und Aspekt-Systemen finden wir nur wenige Grammtypen: Perfektiv:Imperfektiv, Präteritum („Past”) und Futur. Perfektiv:Imperfektiv ist in strukturalistischer Terminologie ausgedrückt eine „äquipollente Opposition”, wo beide Glieder markiert sein können.

Diese Opposition hat nicht nur eine aspektuelle, sondern auch eine temporale Bedeutung: Das Perfektiv wird allgemein auf die Vergangenheitsreferenz beschränkt. Der Grammtyp Präteritum wird oft nur mit dem Imperfektiv kombiniert (das "Imperfekt” der traditionellen Grammatik). Vier Arten von Realisationen der „Kerntypen” Perfektiv: Imperfektiv und Präteritum treten häufig auf. (Figur 2). 
Typ 0: Keine Kemgramme (gowohnlich)
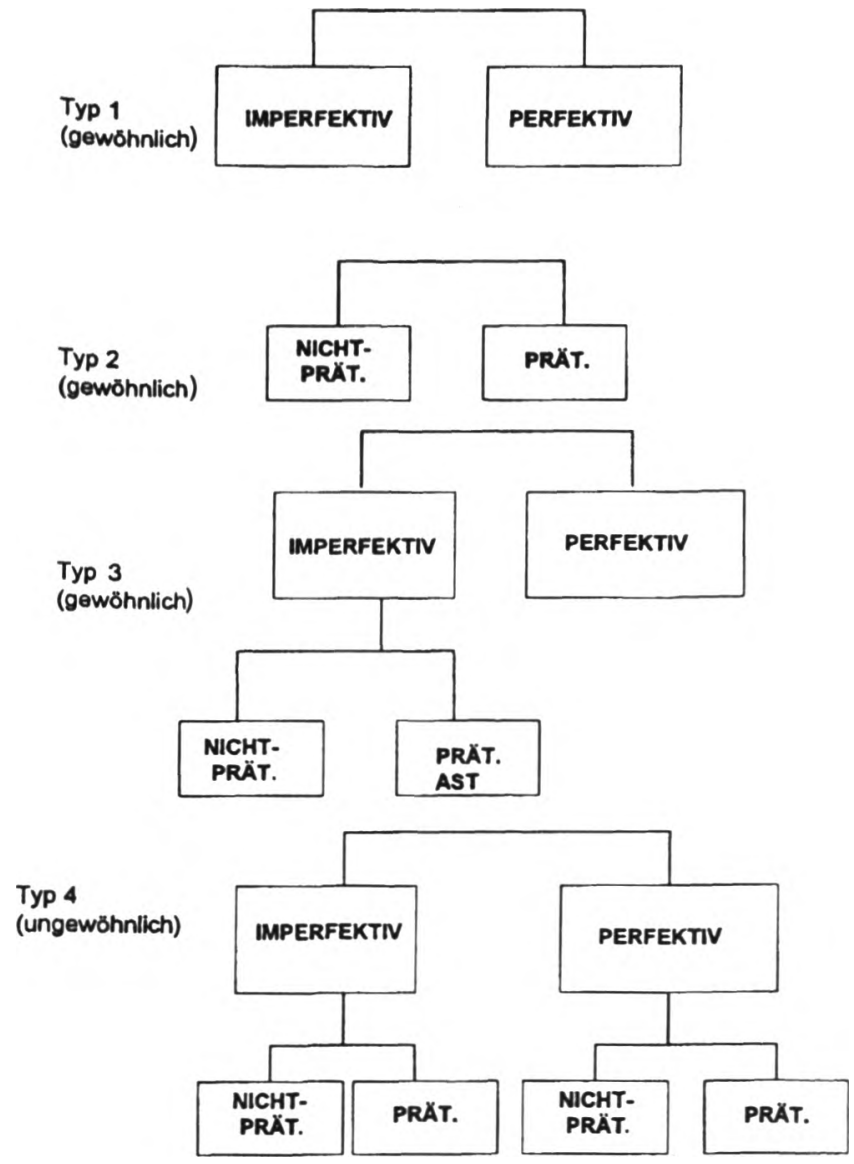

Figur 2. Systeme von Kerngrammen

An der Peripherie des Systems finden wir Grammtypen wie das Perfekt, das Progressiv und die ,jüngeren" Zukunftskonstruktionen (hier „Futuroiden” genannt), die alle normalerweise periphrastich (analytisch) ausgedrückt werden. Außerhalb des Systems sind einige der typischen lexikalischen Quellen der verschiedenen Grammtypen angegeben. 


\section{Areale Tendenzen}

Man kann in der Arealtypologie Erscheinungen auf wenigstens zwei Ebenen unterscheiden, die man die Mikro-Ebene und die Makro-Ebene nennen kann.

Die Mikro-Ebene ist das traditionelle Forschungsgebiet der arealen Linguistik, in der der Begriff „Sprachbund” zentral gewesen ist. Ein Sprachbund (das Wort wird auch in anderen Sprachen gebraucht) ist eine Gruppe von Sprachen, die geographisch benachbart, aber nicht notwendigerweise genetisch verwandt sind und die ähnliche grammatische Entwicklungen aufweisen. Man kann wohl sagen, daB Sprachbundphänomene in Grammatikalisierungsprozessen eher die Regel als eine Ausnahme sind - solche Prozesse verbreiten sich oft zu mehreren benachbarten Sprachen und schaffen dadurch Grammfamilien. Beispiele von solchen Grammfamilien finden wir in Figur 3, wo die wichtigsten Futurkonstruktionen in den europäischen Sprachen gezeigt werden.

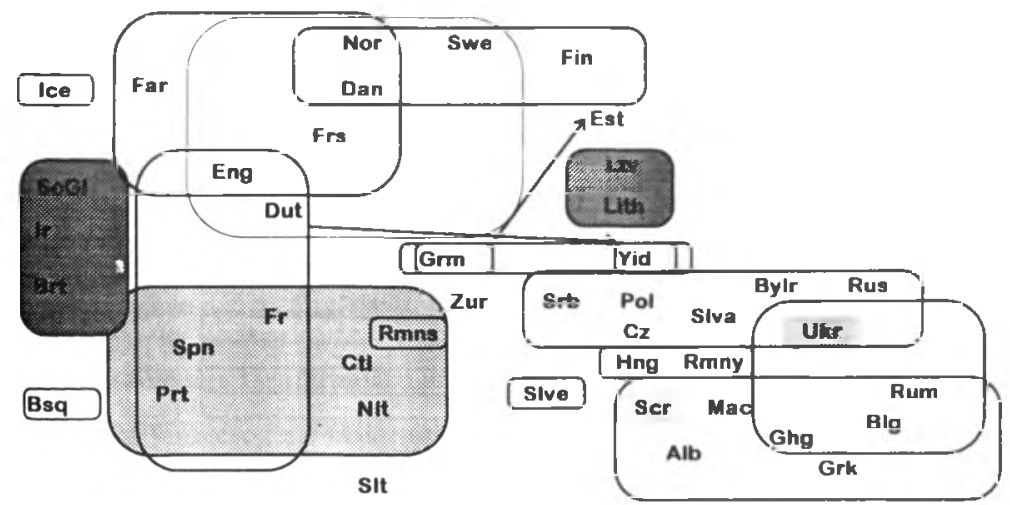

Figur 3. Grammfamilien in Europa, die Zukunftsreferenz ausdrücken. Synthetisch ausgedrückte Gramme sind grau grundiert.

Die Makro-Ebene behandelt statistische Tendenzen, die für Kontinente oder Teile von Kontinenten gelten. In der typologischen Forschung ist es jetzt klar geworden, daß die meisten grammatischen Phänomene geographisch nicht gleichmäBig verteilt sind. Das gilt z.B. für die Wortfolgetypen von Greenberg (SOV, SVO usw.), wie in den letzten Jahren gezeigt worden ist. Tempus- und Aspektsysteme sind in dieser Hinsicht keine Ausnahme. Die Grammtypen verteilen sich geographisch sehr ungleichmäßig: die arealen Einflüsse sind im Bereich der Grammatikalisierungsprozesse sehr stark. Letzten Endes sind natürlich die arealen Makro-Erscheinungen auf die Mikro-Ebene zurückzuführen. 
Figur 4 zeigt die Verteilung der Grammtypen Präteritum und Imperfektiv/Perfektiv in der Sprachenauswahl von Bybee et al. (1994). (Die Karte ist nicht skalenrecht.) Man sieht hier deutlich die Konzentrationen von Sprachen bestimmter Typen. Der Grammtyp Präteritum hat dabei eine besonders deutliche Verbreitung.

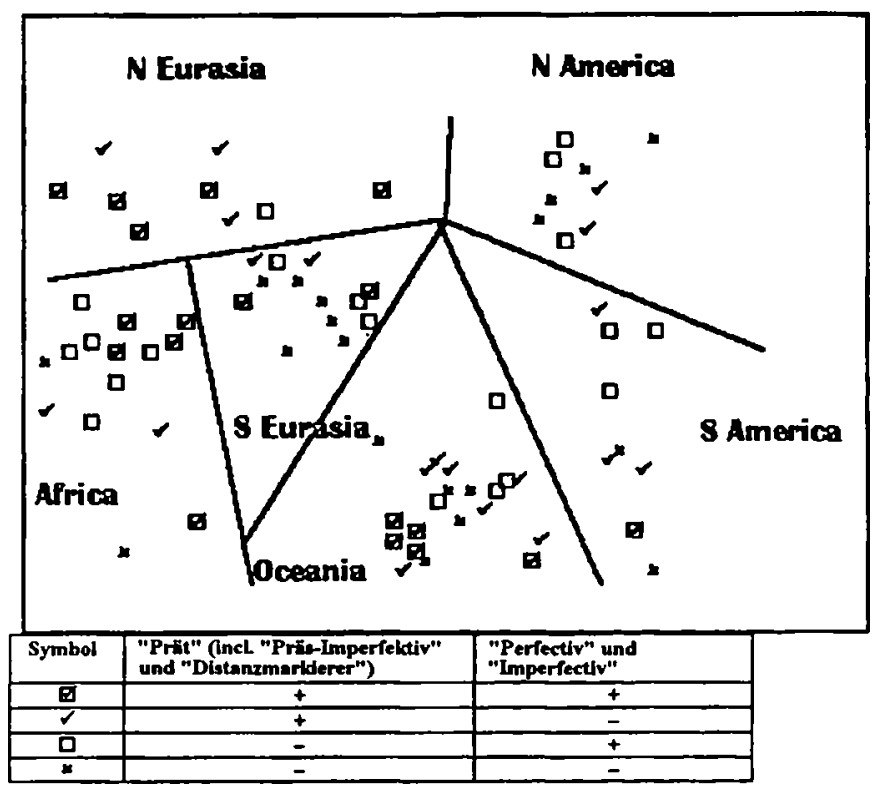

Figur 4. Verteilung der Hauptgrammtypen in der Auswahl von Bybee et al. 1994

In den europäischen Sprachen finden wir ganz bestimmte areale Tendenzen:

- Alle europäische Sprachen haben den Grammtyp Präteritum.

- Der Unterschied Perfektiv:Imperfektiv fehlt in vielen Sprachen, besonders in Nord-Europa.

- Das Perfekt kommt in den europäischen Sprachen sehr häufig vor; das „habeo-Perfekt” - d.h. ein Perfekt das auf einer possessiven Konstruktion basiert - findet man fast nur in diesem Weltteil. 
- In Nord-Europa ist auBerdem das Futur nur schwach grammatikalisiert oder fehlt überhaupt.

Für das Verstehen des deutschen Systems sind die zwei letzten Punkte von besonderer Bedeutung, und wir werden sie jetzt näher beleuchten.

\section{Das Perfekt in Europa}

Das Perfekt kommt in den europäischen Sprachen sehr häufig vor. Das "habeo-Perfekt" findet man fast nur in diesem Weltteil. In vielen Sprachen, z.B. im Deutschen, ist das Perfekt eine Mischung zwischen dem "habeo" und dem „esse"-Typ.

Das Perfekt ist in den peripheren Teilen Europas gut bewahrt.

Die Expansion des alten Perfekts in mehreren Sprachen des europäischen Zentralgebietes, auch im Deutschen, und der damit zusammenhängende "Präteritumschwund" in vielen Varianten des Deutschen ist ein klassischer Fall von Grammatikalisierung. Weil dieser Prozeb nicht überall gleichmäßig weit gegangen ist, bietet das „Kontinentalgermanische” eine Möglichkeit, die Grammatikalisierung „live” zu studieren.

Über die Semantik der Vergangenheitstempora im Deutschen ist sehr viel geschrieben worden. Die Literatur gibt einen Eindruck von Verwirrung, teils sogar von Verzweiflung.

Aus typologischer Sicht muß man die Situation im Hochdeutschen als ein typisches Übergangssystem charakterisieren. Solche Systeme lassen sich schlecht an das strukturalistische Ideal anpassen, aber sind aus der Perspektive der Grammatikalisierungstheorie heraus normal.

Die nicht vollgezogene Grammatikalisierung des Perfekts zu einem allgemeinen Präteritumtempus schafft eine eigenartige Konkurrenzsituation zwischen dem Perfekt und dem alten Präteritum, wo es eigentlich nicht möglich ist, die Wahl zwischen den beiden Grammen in einer einfachen Formel zu erfassen. Am besten können wir eine Reihe von Faktoren identifizieren, die die Wahl beeinflussen.

Latzel (1974), (1977) hat die Wahl zwischen den deutschen Vergangenheitstempora quantitativ studiert. Am interessantesten für unsere Zwecke sind vielleicht seine Daten über den Gebrauch von Perfekt und Präteritum in Todesanzeigen in verschiedenen Teilen Deutschlands, d.h. wo der Kontext konstant gehalten und die geographische Dimension variiert wird: 


$\begin{array}{lll} & \text { Perfekt (\%) } & \text { Präteritum (\%) } \\ \text { Süddeutsches Sprachgebiet } & 56 & 44 \\ \text { Rhein-Main-Gebiet } & 39 & 61 \\ \text { Niederdeutsches Sprachgebiet } & 29 & 81\end{array}$

Die Entwicklung von Perfekt zu Präteritum folgt in allgemeinen einem bestimmten Muster, wobei die letzten Kontexte, die davon berührt werden, die erzählenden/narrativen sind. Die Entwicklung im Deutschen ist ein gutes Beispiel dafür. DaB das Präteritum das bevorzugte Erzählungstempus im Deutschen ist, ist eine wohlgekannte Tatsache, die unter anderem der Ausgangspunkt der Tempus- und Aspekttheorie von H. Weinrich (1964) war. Nach der Meinung von Weinrich ist das Präteritum ein Tempus der „erzählten Welt”. Eine Variation desselben Themas ist die Unterscheidung zwischen dem Perfekt als ein "Isolations-Tempus" und dem Präteritum als ein „Ketten-Tempus”. Latzel (1974) kommt zu dem SchluB, daß es im Deutschen besondere Beschränkungen für den Gebrauch von Präteritum in Ein-Satz-Texten und in Einleitungssätzen zu längeren Texten gibt. Man könnte also eine Todesanzeige wie (a) oder (b) aber nicht wie (c) beginnen:
(a) Am ... starb unser Mitarbeiter Herr $X Y$
(b) Unser Mitarbeiter Herr $X Y$ ist gestorben
(c) Unser Mitarbeiter Herr XY starb

Mehrere Forscher haben vorgeschlagen, Fälle des „echten Perfekts” von Fällen des "Quasiperfekts" (mit der Terminologie von Fabricius-Hansen 1986) zu unterscheiden, wobei das „echte Perfekt” etwa dem englischen Perfekt entspricht. In einer extremen Version findet man diese These in Bäuerle (1979), wo behauptet wird, daß das deutsche Perfekt homonym sei, d.h. daß man es als zwei verschiedene Einheiten sehen muß, wobei die eine einfach eine von zwei Realisierungsmöglichkeiten der Kategorie Präteritum sei. Die Homonymiethese scheint nicht mit dem KontinuumCharakter der Unterscheidung zwischen den Vergangenheitstempora vereinbar zu sein. Die Unterscheidung von „echten” und "unechten” Fällen des Perfekts ist dagegen ganz kompatibel mit der Hypothese, daB das deutsche Perfekt sich in einem Übergangsstadium zwischen einem (typischen) Perfekt und einem allgemeinen Präteritum befindet.

Die germanischen Sprachen, in denen das Perfekt das Präteritum verdrängt hat, gliedern sich in zwei Typen. Im ersten (z.B. Afrikaans) ist die Präteritumform der Kopula (was/war) noch bewahrt. Im zweiten (z.B. Jiddisch) ist diese Form verlorengegangen: man verwendet auch hier das Perfekt. 
Im deutschen Sprachraum findet man Beispiele beider Typen: der erste im Süd-Osten, der zweite im Süd-Westen.

\section{Die Zukunft im Deutschen}

Die Zukunftsreferenz wird im Deutschen, besonders in der gesprochenen Sprache, verhältnismäBig oft nicht markiert. Statt dessen verwendet man das Präsens. Die werden-Konstruktion ist historisch gesehen relativ jung und ist noch nicht in alle Dialekte eingedrungen; so wird sie z.B. im Zürichdeutschen als fremd empfunden (Bickel 1992). In diesem Sinn ist das Deutsche ein Teil des "nordeuropäischen futurlosen Gebietes", das alle germanischen Sprachen (ausgenommen das Englische) und auch die Ostseefinnischen (Finnisch, Estnisch) umfaBt. Was diese Sprachen im Vergleich mit etwa dem Englischen und dem Französichen charakterisiert, ist die Abwesenheit einer grammatikalisierten, d.h. obligatorischen Markierung von Zukunftsreferenz in "reinen Voraussagen”. Im folgenden Satz aus unserem EUROTYP-Fragebogen wurden für das Deutsche sowohl das Präsens als auch die periphrastische Konstruktion mit werden ("Futur I") als Alternative gegeben: Morgen regnet es/wird es regnen. Im Englischen wäre es dagegen nicht möglich, das Präsens zu verwenden: It's going to rain/will rain tomorrow.

Das futurlose Gebiet war früher stärker ausgeprägt als heute: sowohl das Gotische wie auch das Altslawische hatten eigentlich keine systematischen, grammatischen Mittel, die Zukunft zu markieren.

\section{Die Armut des Deutschen}

In meinem Vortrag habe ich ein wenig provokativ gesagt, daB das deutsche Tempus- und Aspektsystem im allgemeinen recht arm ist. Das würde vor allem für die Dialekte gelten, wo der Präteritumschwund völlig durchgeführt worden ist und auch das werden-Futur nicht systematisch gebraucht wird. Man vergleiche z.B. das Bulgarische, wo ein deutscher Satz wie Er hat einen Brief geschrieben wenigstens zwölf Übersetzungen hat.

Dabei sollte man wohl hinzufügen, daß diese "Armut" nicht bedeutet, $\mathrm{da} B$ die deutsche Sprache weniger ausdrucksfähig ist. Es handelt sich ja hier darum, was grammatisch markiert wird, nicht um die allgemeine Ausdruckskraft der Sprache. 


\section{Literatur}

Bāuerle, Rainer (1979): Temporale Deixis - temporale Frage. Tübingen.

Bybee, Joan L. (1985): Morphology: a study of the relation between meaning and form. Amsterdam.

Bybee, Joan/Dahl, Ōsten (1989): The creation of tense and aspect systems in the languages of the world. In: Studies in Language 13, S. 51-103.

Bybee, Joan/Perkins, Revere/Pagliuca, William (1994): The Grammaticization of Tense, Aspect and Modality in the Languages of the World. Chicago.

Bickel, Balthasar (1992): The Marking of Future Time Reference in Züritūütsch. Future Time Reference in European Languages I. (EUROTYP Working Papers VI.2).

Chung, Sandra/Timberlake, Alan (1985): Tense, Aspect, and Mood. In: Shopen, Timothy (Hg.): Language Typology and Syntactic Description, I: Clause Structure. Cambridge. S. 202-258.

Comrie, B. (1976): Aspect. An Introduction to the Study of Verbal Aspect and Related Problems. Cambridge.

Comrie, B. (1985): Tense. Cambridge.

Csató, Eva (1992): On some theoretical and methodological problems of the typological study of tense-aspect categories. (EUROTYP Working Papers, Series VI, No. 1).

Dahl, Östen (1985): Tense and Aspect Systems. Oxford.

Fabricius-Hansen, Cathrine (1986): Tempus fugit. Düsseldorf.

Friedrich, P. (1974): On aspect theory and Homeric aspect. In: International Journal of American Linguistics. vol. 40, No. 4., Part 2, Memoir 28.

Gvozdanović, Jadranka/Janssen, Theo A.J.M., (Hg.) (1991): The function of tense in texts. Amsterdam.

Latzel, Sigbert (1974): Perfekt und Präteritum im Ein-Satz-Āusserungen der geschriebenen deutschen Sprache. In: Gelhaus, Hermann/Latzel, Sigbert (Hg.): Studien zum Tempusgebrauch im Deutschen. Tübingen. S. 173-274.

Latzel, Sigbert (1977): Die deutschen Tempora Perfekt und Präteritum. München.

Ultan, Russell (1978): The nature of future tenses. In: Greenberg, J. ( $\mathrm{Hg}$.): Universals of human language. Vol. 3. Word structure. Stanford. S. 82123.

Weinrich, Harald (1964): Tempus - besprochene und erzählte Welt. 2. Aufl. Stuttgart. 